\title{
Nano shell impact on Huygens' metasurface dipolar resonances and optical response
}

\author{
Hasan Kocer, ${ }^{1, *}$ (D) Halil IsIK ${ }_{9}{ }^{1}$ Yilmaz Durna, ${ }^{1}$ Bahram Khalichi, ${ }^{1,2}$ (D) Hamza Kurt ${ }^{3}{ }^{3}$ And \\ EKMEL OZBAY ${ }^{1,2,4,5,6}$ \\ ${ }^{1}$ NANOTAM-Nanotechnology Research Center, Bilkent University, 06800 Ankara, Turkey \\ ${ }^{2}$ Department of Electrical and Electronics Engineering, Bilkent University, 06800 Ankara, Turkey \\ ${ }^{3}$ School of Electrical Engineering, Korea Advanced Institute of Science and Technology (KAIST), Daejeon, Republic of Korea \\ ${ }^{4}$ Department of Physics, Bilkent University, 06800 Ankara, Turkey \\ ${ }^{5}$ UNAM-Institute of Materials Science and Nanotechnology, Bilkent University, 06800 Ankara, Turkey \\ ${ }^{6}$ e-mail: ozbay@bilkent.edu.tr \\ *Corresponding author: hasan.kocer@bilkent.edu.tr
}

Received 10 March 2021; revised 15 June 2021; accepted 8 July 2021; posted 8 July 2021 (Doc. ID 424589 ); published 6 August 2021

\begin{abstract}
Due to several advantages over conventional devices for the control of electromagnetic (EM) radiation, the demand for metasurface utilization based on artificially engineered micro and nanostructures is boosted, especially in new generation devices. Among the metasurfaces family, there has been a growing interest in Huygens' metasurfaces that are easy to fabricate due to their lower aspect ratio compared to their counterparts and also provide alternative electromagnetic radiation control by tuning the dipolar electric and magnetic resonances. In this study, an all-dielectric Huygens' metasurface consisting of the high-refractive-index nano shells embedded in the low-refractive-index environment is designed and extensively investigated numerically and analytically in the nearinfrared spectrum. By simply tuning the nano shell inner radius, the effects on the dipolar resonances are unveiled specific to the proposed design. To assess the EM wave interactions in the designed Huygens' metasurface, an analytical model based on the coupled discrete dipole approach is applied for selected distinct cases of the designed metasurface. It is shown that the spectral position of the dipolar resonances can be detuned or tuned simultaneously depending on the structural parameter of the meta-atoms arranged in a periodic array. This study sheds light on the physics and abilities of the nano shell structure as a Huygens' metasurface for the potential applications of metasurface-based light-matter interaction including imaging and sensing. ( 2021 Optical Society of America
\end{abstract}

https://doi.org/10.1364/JOSAB.424589

\section{INTRODUCTION}

Metasurfaces, the two-dimensional equivalent of metamaterials where limitations are relaxed due to their subwavelength thickness, have attracted considerable research interest in the last decade [1-4]. Although plasmonic structures [5,6] were widely researched at the beginning, due to their high ohmic losses, the direction of the research has recently moved to dielectric metasurfaces $[7,8]$. All dielectric metasurfaces offer high efficiency and low loss, especially in the transmission of the light. Such structures are studied experimentally and theoretically with a wide range of different approaches [7,9-13]. The most salient of these approaches can be classified as transmissive Pancharatnam-Berry (PB) metasurfaces [14-18], high-contrast metasurfaces [19-23], and Huygens' metasurfaces $[3,4,8,12,24-27]$. While PB metasurfaces can only work with circularly polarized light, the high-contrast ones show fabrication difficulties due to the high aspect ratio. The last approach, Huygens' metasurfaces, offers ease of fabrication as they have a low aspect ratio. The concept of Huygens' metasurfaces is based on the surface equivalence theorem [28,29] and the corresponding dielectric Mie resonators [27,30,31]. The resonating behavior is considered as a basic building block of Huygens' optical metasurfaces. Due to the dielectric nature of these resonators, they show almost lossless properties. In addition, the scattering patterns of these structures in the visible and near-infrared ranges can vary according to the size, shape, and composition. Here, meta-atoms have dipolar resonances such as an electric dipole (ED) and a magnetic dipole (MD). The control of the propagation of light can be obtained in an interesting manner by the interaction of the radiations scattered in the forward and backward directions from these dipoles and the interference of the dipoles with each other. Essentially, they support both strong electrical and magnetic responses (optical magnetism without magnetic material) such that the overlapping of these responses leads to zero backscattering, which is known as the Kerker condition [32-34]. In addition, by controlling the interaction between the two resonance modes, the 
transmission spectrum of the incident light can be manipulated at certain wavelengths such that a near-unity reflectivity or complete transmission can be realized.

Experimental and theoretical studies were conducted on Huygens' metasurfaces consisting of meta-atoms made of highrefractive-index nano discs in low-refractive-index environment in order to tune $\mathrm{ED}$ and $\mathrm{MD}$ resonances in the optical spectrum $[3,24,26,35]$. In these studies, the dimensions (diameter and height of cylindrical disc) and the lattice period [36] of the nano disc geometry were mostly investigated and optimized. In other words, the nano disc structure is considered as a solid element in the traditional Huygens' metasurface. Although the nano shell structure may seem simple, it contains richness worth investigating in light-matter interaction due to the formation of different electromagnetic boundary conditions compared to the pure solid body in traditional Huygens nanoarchitectures. Therefore, research still needs to be conducted on how the behavior of $\mathrm{ED}$ and MD resonances are affected when the nanostructure is designed as a nano shell under the constant lattice period [37].

In this study, in order to unveil the effect of the dipolar resonances in a Huygens' metasurface with hollow nano cylinders, numerical and analytical studies are performed with a periodic array of a high-index and lossless nano shell structure buried in a low-index material in the near-infrared region. The overlap of resonances and their spacing with respect to each other can be controlled by changing only the filling ratio of the meta-atom while keeping the aspect ratio and periodicity of the array the same. Moreover, the localization of the light at certain regions can also be succeeded due to the specific configuration of the metasurfaces building block. Such a feature is desired for sensing applications.

\section{DESIGN AND SIMULATION RESULTS}

The schematic of the proposed metasurface design is presented in Fig. 1. It consists of a periodic array of high-index silicon (Si) nano shells embedded in a low-index homogeneous silicon dioxide $\left(\mathrm{SiO}_{2}\right)$ environment. Figure 1(a) not only shows the structure to be used in the numerical simulations, but also describes the waves scattered from the meta-atoms in the analytical approach to be mentioned later. Since the wavelength range used in the study is $1.1-1.8 \mu \mathrm{m}$, the materials in this range are considered to be lossless. Therefore, the refractive indices for $\mathrm{Si}$ and $\mathrm{SiO}_{2}$ are taken as constant 3.5 and 1.5, respectively. Numerical electromagnetic simulations are performed with Lumerical FDTD, a commercially available finite-difference time-domain (FDTD) simulation software package. The normally incident and $x$-polarized plane wave emitted from the source in the $+z$ direction $\left(\vec{E}_{\text {inc }}\right.$ and $\left.\vec{k}_{\text {inc }}\right)$ interacts with the metasurface and causes radiation to scatter from the metasurface in the forward $\left(\vec{E}_{f}\right.$ and $\left.\vec{k}_{f}\right)$ and backward $\left(\vec{E}_{r}\right.$ and $\left.\vec{k}_{r}\right)$ directions. In the simulations, perfectly matched layers along the $z$ axis and the periodic boundary conditions along the $x$ and $y$ axes are selected. The lattice constants $(\Lambda)$ along the $x$ and $y$ axes are set to a fixed value of $730 \mathrm{~nm}$. The rest of the geometrical parameters are seen in Fig. 1(b). The height $(b)$ and the outer radius $\left(r_{\text {outer }}\right)$ of the nano shell are kept at constant values of 220 and $325 \mathrm{~nm}$, while the inner radius $\left(r_{\text {inner }}\right)$ is changed from 0

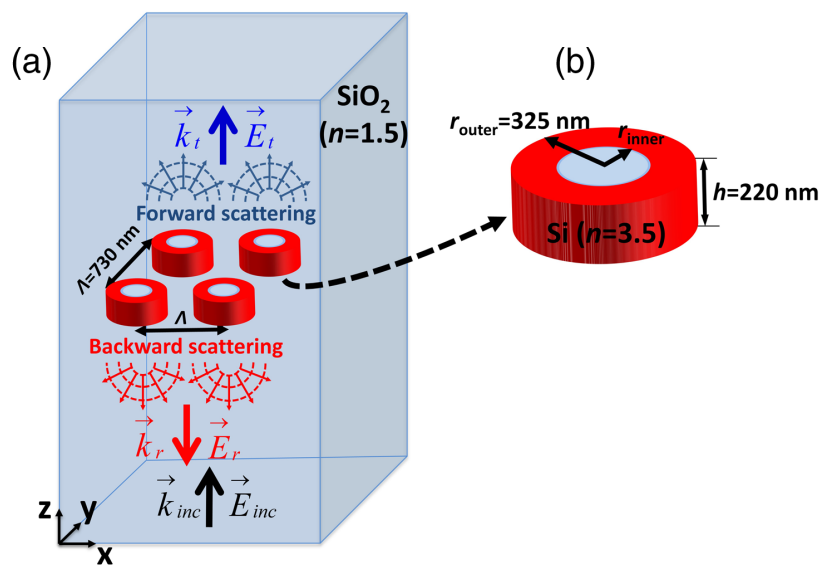

Fig. 1. (a) Schematic of a periodic silicon nano shell array showing the interaction between light and the Huygens' metasurface. The metasurface array is embedded inside a homogeneous low-index medium $\left(\mathrm{SiO}_{2}\right)$. (b) 3D view of the unit nano shell structure displaying the geometrical and material parameters. The structure up to radius $r_{\text {inner }}$ is filled with $\mathrm{SiO}_{2}$.

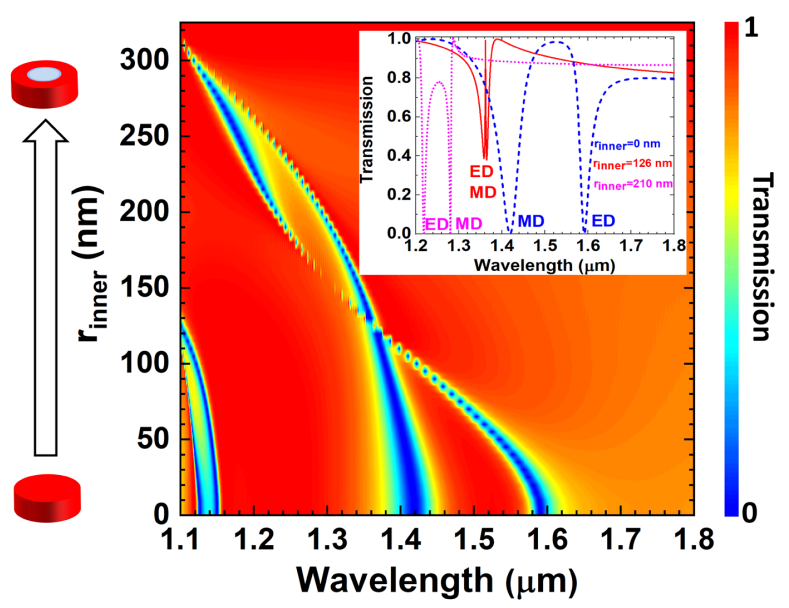

Fig. 2. Spectral transmission of the Huygens' metasurface array with respect to the inner radius of the nano shell. Inset: transmission spectrum when the inner radius is set to $0 \mathrm{~nm}$ (blue dashed line), $126 \mathrm{~nm}$ (red solid line), and $210 \mathrm{~nm}$ (magenta dotted line). ED, electric dipole resonance; $\mathrm{MD}$, magnetic dipole resonance.

(solid disc) to $r_{\text {outer }}$ where the nano shell disappears. The determination of fixed parameters is inspired by similar parameters in the experimental and numerical studies of solid nano discs $[3,24,26]$.

Under the aforementioned material parameters and simulation conditions, Fig. 2 presents the numerically obtained transmission spectrum while the inner radius of the design is changing. The bluish spiral curve in the middle of the $2 \mathrm{D}$ color map shows the behavior of the ED and MD resonances peculiar to the nano shell structure. In the initial state where the inner radius is zero, the shell architecture is in the form of a solid disk. The inset in the figure depicts this case, hereinafter referred to as "Case A," in the line graph (blue dashed line). As can be seen in the graph, in this case the ED and MD resonances are separate, and the ED resonance wavelength is larger than the MD resonance wavelength. This determination is consistent 


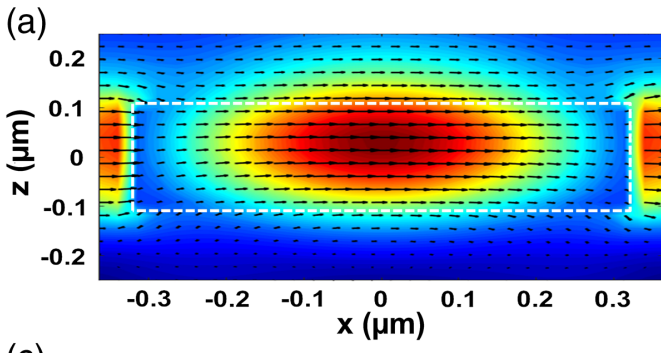

(c)

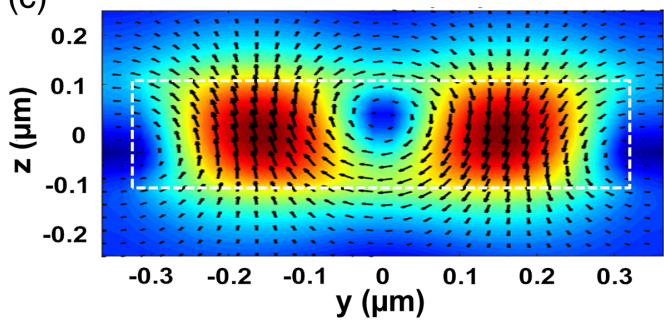

(b)

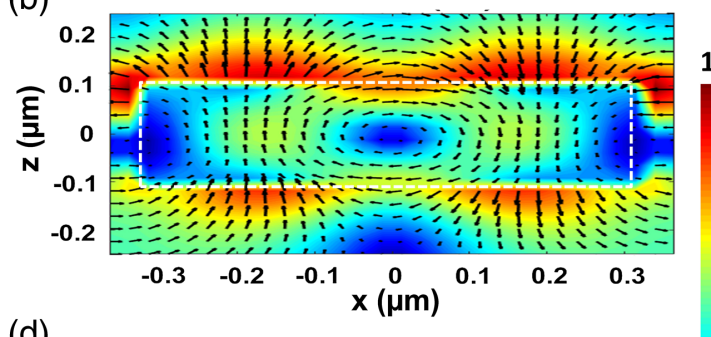

(d)

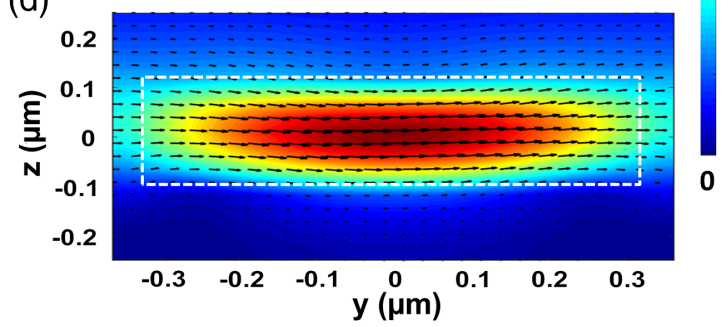

Fig. 3. Normalized electric field distributions in the central $x-z$ plane (a) at ED and (b) at MD resonances, and the normalized magnetic field distributions in the central $y-z$ plane (c) at ED and (d) at MD resonances for Case $\mathrm{A}\left(r_{\text {inner }}=0 \mathrm{~nm}\right)$. The dashed white line in all of the panels represents the boundary of the nano shell. Black arrows are vector distributions of the field of interest ( $E$-field or $H$-field). Note that the dipolar resonance wavelengths are $\lambda_{\mathrm{ED}}=1591 \mathrm{~nm}$ and $\lambda_{\mathrm{MD}}=1418 \mathrm{~nm}$ for this case.

with a recent study [24] that explains the interaction of near-IR light with a similar Huygens' metasurface consisting of only a solid nano disc. Looking back at Fig. 2, it is seen that, when the inner radius is incremented (by replacing silicon with silica and keeping the outer radius constant), the ED and MD resonances begin to come close to each other. With the increase in the inner radius, the shift in the $\mathrm{ED}$ resonance wavelength is stronger than the MD. Moreover, the linewidths are not the same and ED resonance has a smaller linewidth. The condition known as the Kerker effect [32] in which both resonances overlap, hereinafter referred to as "Case B" in this study, occurs at $r_{\text {inner }}=126 \mathrm{~nm}$. The transmission spectrum of Case B in the inset is given as a red solid line between 1.2 and $1.8 \mu \mathrm{m}$ wavelengths. As can be clearly seen here, the transmission in this state (i.e., Case B) is greatly increased and even an almost perfect transmission is obtained at a wavelength of approximately $1.36 \mu \mathrm{m}$. When the inner radius in Case $\mathrm{B}$ is exceeded, the ED and MD resonances begin to decompose again, but interestingly, their positions in the spectrum with respect to Case A are reversed. In other words, the $\mathrm{ED}$ resonance wavelength is smaller than the $\mathrm{MD}$ resonance wavelength in contrast to Case A. As observed in the spiral structure, while resonances continue to detour from each other at radii after $126 \mathrm{~nm}$, they become the furthest at a certain radius. This radius is $r_{\text {inner }}=210 \mathrm{~nm}$ and it is called "Case C" in this study. Its transmission line plot is given as a magenta dotted line in the inset. Another remarkable feature of the Case $\mathrm{C}$ is related to the full width at half-maximum (FWHM) of the ED and MD dipolar resonances. The FWHM values for Case $\mathrm{C}$ are narrower with respect to the ones in Case A. That is, sharper discrete dipolar resonances are observed for radii on the other side of the Kerker point. Finally, in the range $210 \mathrm{~nm}<r_{\text {inner }}<325 \mathrm{~nm}$, the dipolar resonances gradually become close to each other and then merge.

In order to unveil the type and character of dipolar resonances in the different situations mentioned hereinabove, the field distributions on the orthogonal planes cutting the nano shell
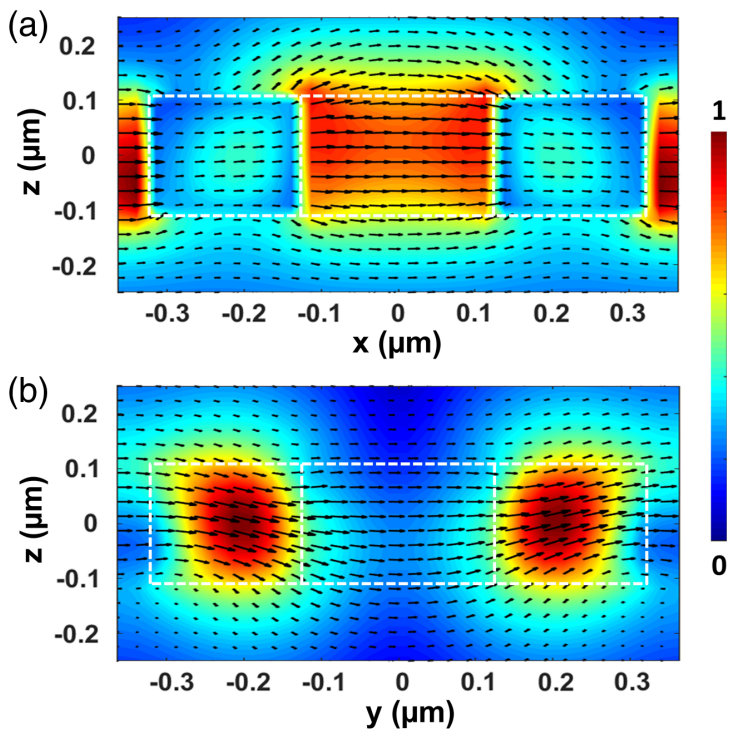

Fig. 4. (a) Normalized electric field distribution in the central $x-z$ plane and (b) normalized magnetic field distribution in the central $y-z$ plane at $\mathrm{ED}$ and $\mathrm{MD}$ overlap resonance for Case $\mathrm{B}$ $\left(r_{\text {inner }}=126 \mathrm{~nm}\right)$. The dashed white line in both panels represents the boundary of the nano shell. The black arrows are vector distributions of the field of interest ( $E$ - field or $H$-field). Note that the dipolar resonance wavelengths are $\lambda_{\mathrm{ED}}=\lambda_{\mathrm{MD}}=1361 \mathrm{~nm}$ for this case.

perpendicular to the center are obtained numerically for Case $\mathrm{A}$ (Fig. 3), Case B (Fig. 4), and Case C (Fig. 5). Next, the remarkable findings in these figures will be elucidated on a case-by-case basis.

According to the results of Case A presented in Fig. 3, it is clearly seen that the occurrence of ED and MD resonances is confirmed by the field distributions. While the electric field in the $x-z$ plane [Fig. 3(a)] is concentrated in the center of the silicon solid disk, the magnetic field in the $y-z$ plane [Fig. 3(c)] 
(a)

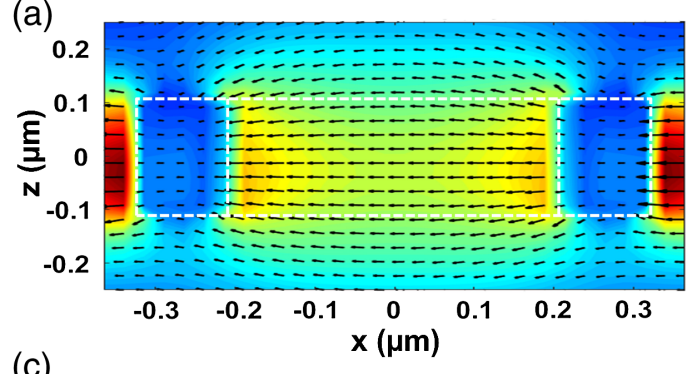

(c)

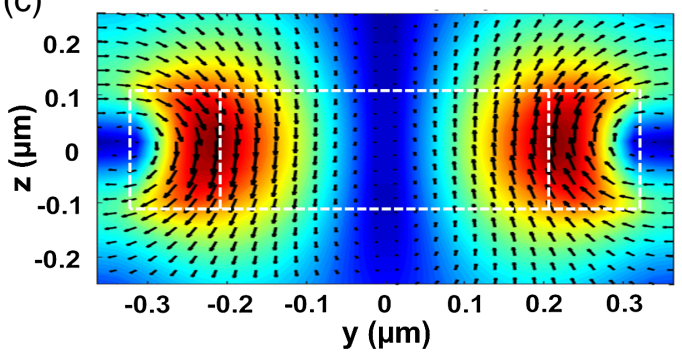

(b)

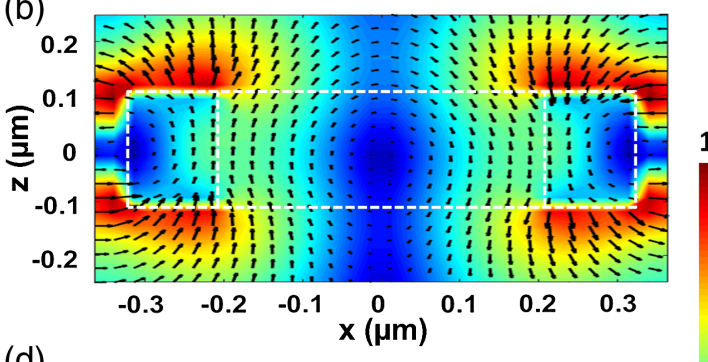

(d)

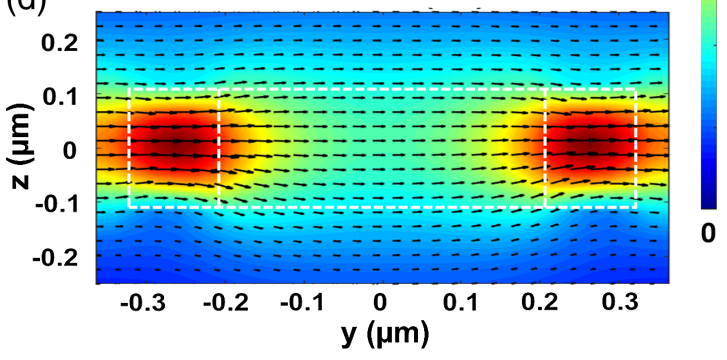

Fig. 5. Normalized electric field distributions in the central $x-z$ plane (a) at $\mathrm{ED}$ and (b) at $\mathrm{MD}$ resonances, and the normalized magnetic field distributions in the central $y-z$ plane (c) at $\mathrm{ED}$ and $(\mathrm{d})$ at $\mathrm{MD}$ resonances for Case $\mathrm{C}\left(r_{\text {inner }}=210 \mathrm{~nm}\right)$. The dashed white line in all of the panels represents the boundary of the nano shell. The black arrows are vector distributions of the field of interest ( $E$-field or $H$-field). Note that the dipolar resonance wavelengths are $\lambda_{\mathrm{ED}}=1218 \mathrm{~nm}$ and $\lambda_{\mathrm{MD}}=1280 \mathrm{~nm}$ for this case.

creates a curl around this electric field. Therefore, it becomes clear in Case A that the behavior at this resonant wavelength $\left(\lambda_{\mathrm{ED}}=1591 \mathrm{~nm}\right)$ is in the character of ED resonance. On the other hand, at the wavelength of $\lambda_{\mathrm{MD}}=1418 \mathrm{~nm}$, the electric and magnetic field behavior reverses. In other words, while the electric field in the $x-z$ plane [Fig. 3(b)] behaves like a vortex, the magnetic field in the $y-z$ plane [Fig. 3(d)] is concentrated in the center of the disk and, therefore, the type of behavior at this resonant wavelength is $\mathrm{MD}$ resonance.

As explained earlier, Case $B$ is a special Kerker-like state where the ED and MD resonances overlap. The results are presented in Fig. 4 at an overlap wavelength of $1361 \mathrm{~nm}$. The fields within the white lines representing the boundary of the nano shell exhibit amazing features. It is observed that the electric field in the $x-z$ plane is concentrated in the low-refractive-index $\mathrm{SiO}_{2}$ in the inner region, and the magnetic field in the $y-z$ plane is intensified in the high-refractive-index silicon. Since the refractive index contrast is formed at the $\mathrm{Si} / \mathrm{SiO}_{2}$ interface, discontinuity occurs in the electric field. However, the boundary conditions dictate the continuity of electric displacement field (D) at the interface. Therefore, the electric field normal to this high-index contrast surface is increased in the inner low-index region similar to the principle of light confinement in a slot waveguide [38,39].

Finally, Fig. 5 yields the field distributions for the dipolar resonances in Case $\mathrm{C}$ where the inner shell radius is greater than the one in the Kerker condition. In this case, the magnetic fields [Fig. 5(c)] revolving around the localized electric fields [Fig. 5(a)] at the resonance wavelength of $1218 \mathrm{~nm}$ and the electric fields [Fig. 5(b)] rotating around the localized magnetic fields [Fig. 5(d)] at the resonance wavelength of $1280 \mathrm{~nm}$ reappear similar to the ones in Fig. 4. Therefore, it is proved that the resonance at $1218 \mathrm{~nm}$ wavelength is $\mathrm{ED}$ and the other is the MD type.

\section{THEORETICAL ANALYSIS AND VERIFICATION}

To better comprehend the EM wave interactions in the designed Huygens' metasurface, an analytical model based on the coupled discrete dipole approach $[3,27,30]$ is applied. Each array element as the meta-atom in Fig. 1(a) is optically excited and re-emits the forward $\vec{E}_{\text {scat }}(+z)$ and backward $\vec{E}_{\text {scat }}(-z)$ scattered fields [27]. The total transmitted electric field $\left(\vec{E}_{t}\right)$ as a result of the interference of the electric fields scattered from all elements in the forward direction with each other and with the incoming plane wave $\left(\vec{E}_{\text {inc }}\right)$ can be expressed as $\vec{E}_{t}=\vec{E}_{\text {inc }}+\sum \vec{E}_{\text {scat }}(+z)$. Likewise, the total reflected electric field $\left(\vec{E}_{r}\right)$ is described by $\vec{E}_{r}=\sum \vec{E}_{\text {scat }}(-z)$. The addition symbols here work as many as all the array elements within the metasurface. According to the coupled discrete dipole approach, each array element is represented by a pair of ED and MD. These dipoles in the subwavelength lattice element span across the two-dimensional array and form the Huygens' metasurface. Detailed analytical calculations of Huygens' metasurfaces were implemented in $[3,27]$ utilizing the coupled discrete dipole method. Accordingly, the reflection $(r)$ and the transmission $(t)$ coefficients for the lossless Huygens' metasurface are given by $[3,40]$

$$
\begin{gathered}
t=\frac{E_{t}}{E_{\text {inc }}}=1+\mathrm{ED}_{\text {forward }}+\mathrm{MD}_{\text {forward }}, \\
r=\frac{E_{r}}{E_{\text {inc }}}=\mathrm{ED}_{\text {backward }}+\mathrm{MD}_{\text {backward }},
\end{gathered}
$$

where $E D_{\text {forward }}\left(E D_{\text {backward }}\right)$ is the rate of the radiated electric field in the forward (backward) direction due to the ED modes. Analogously, $\mathrm{MD}_{\text {forward }}\left(\mathrm{MD}_{\text {backward }}\right)$ is the rate of the radiated electric field in the forward (backward) direction due to the MD modes. Note that the magnitude of the incident plane wave is 
(a)

\begin{tabular}{ccccc}
\hline Case & $\begin{array}{c}\lambda_{\text {MD }} \\
(\mathrm{nm})\end{array}$ & $\begin{array}{c}\lambda_{\text {ED }} \\
(\mathrm{nm})\end{array}$ & $\begin{array}{c}\gamma_{\mathrm{e}} \\
(\mathrm{THz})\end{array}$ & $\begin{array}{c}\gamma_{\mathrm{m}} \\
(\mathrm{THz})\end{array}$ \\
\hline $\mathrm{A}$ & 1418 & 1591 & 2.20 & 3.50 \\
$\mathrm{~B}$ & 1361 & 1361 & 2.00 & 0.25 \\
$\mathrm{C}$ & 1280 & 1218 & 1.00 & 0.50 \\
\hline
\end{tabular}

(c)

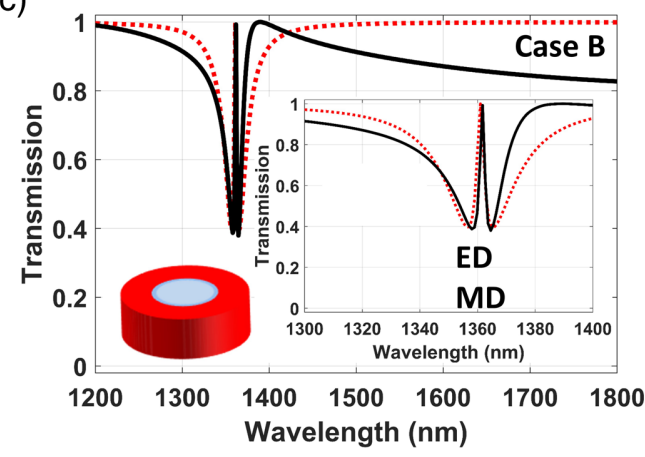

(b)

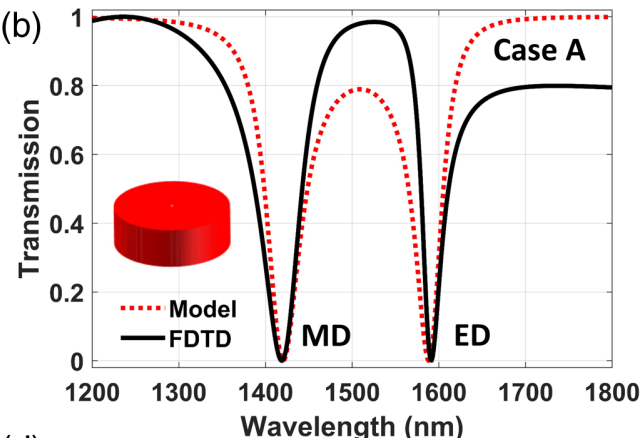

(d)

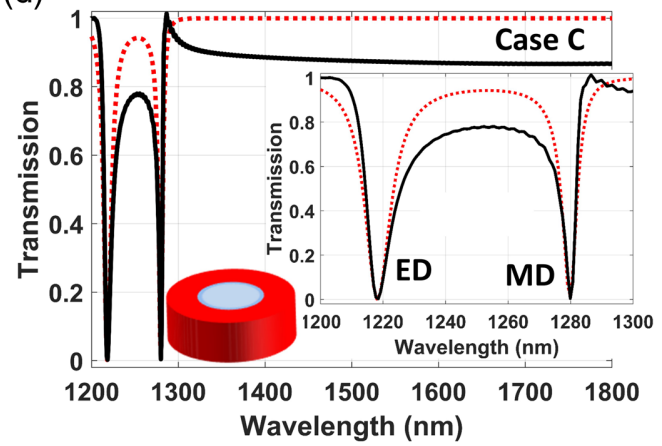

Fig. 6. (a) Model parameters. Analytical model fitting the numerical (FDTD) transmission spectra for (b) Case A, (c) Case B, and (d) Case C. Inset plots in (c) and (d) are zoomed in.

taken as $E_{\mathrm{inc}}=1 \mathrm{~V} / \mathrm{m}$ for simplicity. Due to the ED and MD mode propagation, it can be expressed as $\mathrm{ED}_{\text {forward }}=\mathrm{ED}_{\text {backward }}$ and $\mathrm{MD}_{\text {forward }}=-\mathrm{MD}_{\text {backward }}$. While the sign of the electric fields radiating back and forth from the EDs is the same, the sign of the forward and backward radiating electric fields from the MDs is opposite, a well-known distinguishing feature for ED and $\mathrm{MD}$ radiations $[3,41,42]$. Then, $\mathrm{ED}_{\text {forward }}\left(\mathrm{ED}_{\text {backward }}\right)$ and $M D_{\text {forward }}\left(M_{\text {backward }}\right)$ are expressed based on Lorentzian line shape spectral dependence at $\mathrm{ED}$ and $\mathrm{MD}$ resonances as follows $[3,40]$ :

$$
\begin{gathered}
\mathrm{ED}_{\text {forward }}=\mathrm{ED}_{\text {backward }}=\frac{2 j \gamma_{e} \omega}{\omega_{e}^{2}-\omega^{2}-2 j \gamma_{e} \omega}, \\
\mathrm{MD}_{\text {forward }}=-\mathrm{MD}_{\text {backward }}=\frac{2 j \gamma_{m} \omega}{\omega_{m}^{2}-\omega^{2}-2 j \gamma_{m} \omega},
\end{gathered}
$$

where $\omega_{e}\left(\omega_{m}\right)$ is the resonant angular frequency, and $\gamma_{e}\left(\gamma_{m}\right)$ is the radiative damping parameter at the radiation mode of $\mathrm{ED}(\mathrm{MD}) . \omega$ is the operating angular frequency. Utilizing the transmission and reflection coefficients above, spectral intensity ratios of transmission or transmissivity $(T)$ and reflectivity $(R)$ can be easily obtained as $T=|t|^{2}$ and $R=|r|^{2}$. Although absorptivity $(A)$ is normally expected to be zero in the proposed design due to lossless material components, it is readily acquired as $A=1-R-T$ by the well-known power conservation rule.

The results of the spectral transmission $\left(T=|t|^{2}\right)$ regarding the fitting of the aforementioned analytical model with the FDTD numerical results for three distinct cases by varying the relevant radiative damping parameters in Eqs. (3) and (4) are shown in Fig. 6. In this study, the resonance angular frequencies in these equations (i.e., $\omega_{e}$ and $\omega_{m}$ ) are taken from the numerical spectra (i.e., $\lambda_{\mathrm{ED}}$ and $\lambda_{\mathrm{MD}}$ as the wavelengths in $\mathrm{ED}$ and $\mathrm{MD}$ resonances, respectively). As a result, for each case, the resonance wavelengths and the extracted radiative damping coefficients are presented as a table in Fig. 6(a). In the light of these tabular data, it is evident from the spectral plots in Figs. 6(b)-6(d) that the model is in very good agreement with the FDTD results for all three cases.

The three special cases of the proposed structure, whose characteristic parameters required for modeling have been uncovered, will now be analyzed thoroughly one by one in Figs. 7-9 in order to grasp the operating characteristics specific to each case using only the model parameters. Initially, the analytical results of Case A are taken into consideration in Fig. 7. In this case, where $\mathrm{ED}$ and $\mathrm{MD}$ are detuned, it is evident in Fig. 7 (a) that there is no light transmission but perfect reflection at these wavelengths of the dipolar resonances (i.e., $T=0$ and $R=1$ at $\lambda=1418 \mathrm{~nm}$ and $1591 \mathrm{~nm}$ ). Because the design consists of the lossless materials, it is also analytically seen that the optical power absorption is zero (i.e., $A=0$ ) for all wavelengths of interest in this and the other cases. The physical mechanisms behind the full opaqueness in dipolar resonance wavelengths can be easily revealed from the amplitude [Fig. 7(b)] and phase [Fig. 7(c)] spectra. That is, the amplitude of the electric field forward emitted from only one mode is dominant at these non-overlapping resonance points. To quantify this determination, $E_{\mathrm{ED}} \approx 1 \mathrm{~V} / \mathrm{m}$ and $E_{\mathrm{MD}} \approx 0 \mathrm{~V} / \mathrm{m}$ at ED resonance wavelength of $\lambda=1591 \mathrm{~nm}$ whereas $E_{\mathrm{MD}} \approx 1 \mathrm{~V} / \mathrm{m}$ and $E_{\mathrm{ED}} \approx 0 \mathrm{~V} / \mathrm{m}$ at MD resonance wavelength of $\lambda=1418 \mathrm{~nm}$. However, the phase angles of the electric fields, whose amplitude is about one, are exactly $180^{\circ}$ at the resonance wavelengths. Furthermore, the phase change of the total field is $180^{\circ}$ at the resonances. As a result of the interference of the forwardscattered fields with the incoming radiation $\left(E_{\mathrm{inc}}=1 \mathrm{~V} / \mathrm{m}\right)$ 
(a)

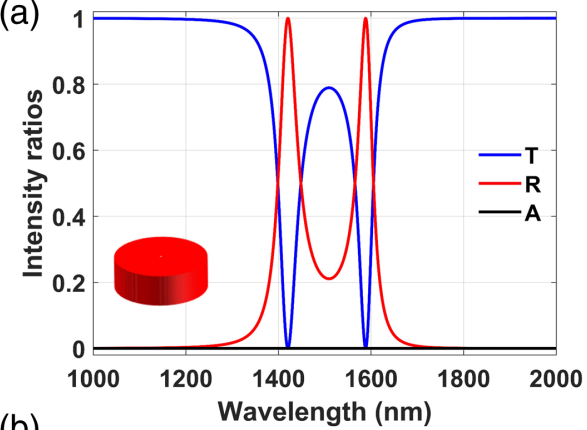

(b)

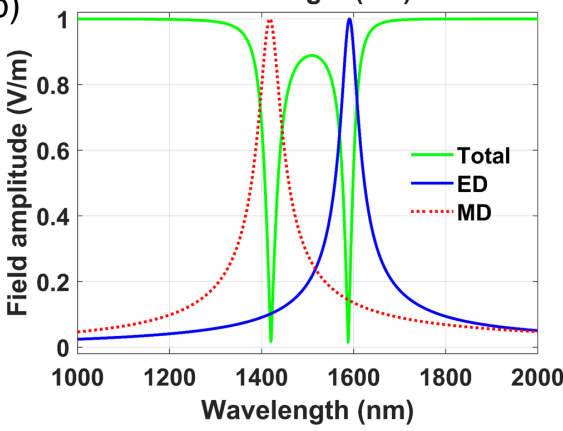

(c)

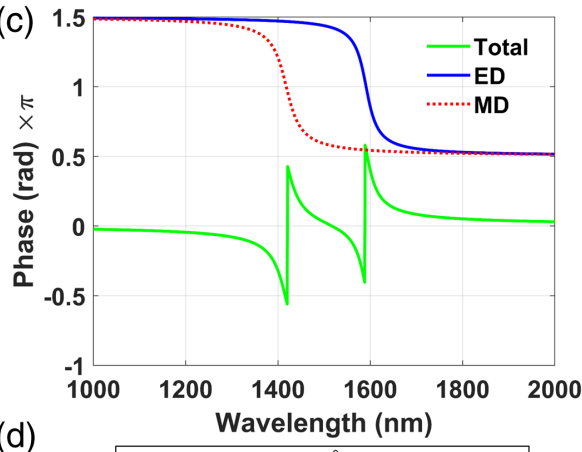

(d)

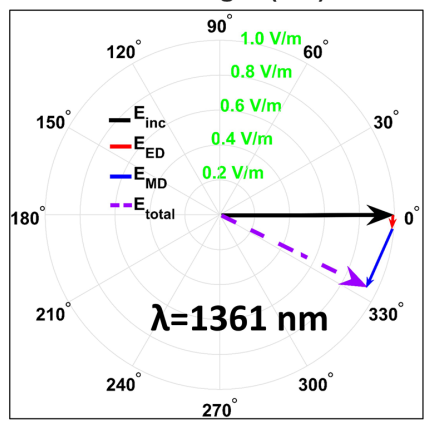

Fig. 7. Analytical results for Case A. (a) Spectral intensity ratios as transmission or transmissivity (blue), reflectivity (red), and absorptivity (black). (b) Electric field amplitude of the transmitted light (green) and the contributions of the electric dipole (blue) and magnetic dipole (red) resonances. (c) Phase spectrum of the transmitted total electric field (green) and the phase spectra of the forward radiated electric field vectors from electric dipole (blue) and magnetic dipole (red) responses. (d) Vector diagram displaying the total output electric field vector $\left(E_{\text {total }}\right)$ formed by the interaction of the incident wave $\left(E_{\mathrm{inc}}\right)$ with the forward radiated electric field vectors from electric dipole $\left(E_{\mathrm{ED}}\right)$ and magnetic dipole $\left(E_{\mathrm{MD}}\right)$ resonances at $\lambda=1361 \mathrm{~nm}$.

at these resonance points, the transmission coefficient is zero and the reflection coefficient becomes -1 at $\mathrm{ED}$ and +1 at $\mathrm{MD}$ resonance. In other words, $E_{\mathrm{E}} / E_{\text {inc }}=\mathrm{ED}_{\text {forward }}=1 e^{(j \pi)}$ and $E_{\mathrm{MD}} / E_{\mathrm{inc}}=\mathrm{MD}_{\text {forward }} \approx 0$ at $\mathrm{ED}$ resonance wavelength $(\lambda=1591 \mathrm{~nm})$, and $\mathrm{MD}_{\text {forward }}=1 e^{(j \pi)}$ and $\mathrm{ED}_{\text {forward }} \approx 0$ at $\mathrm{MD}$ resonance wavelength $(\lambda=1418 \mathrm{~nm})$. Subsequently, if these values are inserted into Eqs. (1) and (2), $t=0$ is obtained at both resonances, while $r=-1$ in $\mathrm{ED}$ and $r=+1$ in MD points. This explains why $T$ is zero and $R$ is one at the discrete dipolar resonances. Figure $7(\mathrm{~d})$ further visualizes the resonance signature in terms of the electric field vector diagram for Case A at a selected wavelength of $\lambda=1361 \mathrm{~nm}$, which is the Kerker point reached at Case B. Note that the total electric field vector is obtained by the addition of the electric field vectors radiating forward from the ED and MD to the incident electric field vector, i.e., the interference of all the transmitted (incoming and scattered) fields at $\lambda=1361 \mathrm{~nm}$.

With the analytical approach discussed in detail hereinabove, the results for Case B are presented in Fig. 8. It can be seen in Fig. 8(a) that, in this case, the transmission is increased and the reflectivity is decreased compared to the previous case, even at $\lambda=1361 \mathrm{~nm}, T=1$, and $R=0$. The underlying reason is that the ED and MD resonances overlap (Kerker condition) and backscattering is blocked due to the destructive interference in the reverse direction where ED and MD modes are out-of-phase, while the forward scattering is strengthened by the constructive interference of the forward-scattered and in-phase dipolar modes. It is possible to explain this argument numerically, for example at $\lambda=1361 \mathrm{~nm}$, by making use of Figs. $8(\mathrm{~b})$ and $8(\mathrm{c}) . \mathrm{ED}_{\text {forward }}=\mathrm{MD}_{\text {forward }}=1 e^{(j \pi)}$, then from Eq. (1), $t=1+1 e^{(j \pi)}+1 e^{(j \pi)}=1-1-1=-1$ (i.e., constructive interference of the dipolar modes). On the other hand, $\mathrm{ED}_{\text {backward }}=\mathrm{ED}_{\text {forward }}=1 e^{(j \pi)}$ and $\mathrm{MD}_{\text {backward }}=-\mathrm{MD}_{\text {forward }}=-1 e^{(j \pi)}$, then from Eq. (2), $r=1 e^{(j \pi)}-1 e^{(j \pi)}=-1-(-1)=0$ (i.e., destructive interference of the dipolar modes). Therefore, $T$ is one and $R$ is zero at $\lambda=1361 \mathrm{~nm}$. Figure 8(d) further illustrates this interference event in terms of the interaction of the incident wave with the forward-scattered electric field vectors from the electric and magnetic dipoles at $\lambda=1361 \mathrm{~nm}$. In addition, two interesting features specific to Huygens' metasurfaces that meet the Kerker condition are easily brought to light by the analytical approach here. One is that, in such cases, where the spectral properties of the dipolar resonances are not exactly the same, the transmission properties are determined by the mode with narrower spectral linewidth (greater quality factor, $Q$ ) This point is clearly seen in the inset of Fig. 8(b) that the spectral features of the narrower MD mode (red colored) are transferred more dominantly to the transmitted light (green). If the two modes have exactly the same spectral shapes, there will be flat and unitary transmission across the spectrum when the Kerker condition is satisfied. $Q$ is proportional to $f_{0} / \gamma$ [43] where $f_{0}$ is the resonant frequency and $\gamma$ is the damping coefficient. From Fig. 6(a), the damping coefficient of the ED mode $\left(\gamma_{e}=2 \mathrm{THz}\right)$ is 8 times higher than the MD mode $\left(\gamma_{m}=0.25 \mathrm{THz}\right)$, which means that the $Q$ of the $\mathrm{MD}$ is 8 times higher than the Qof the ED at the resonant wavelength $\lambda=1361 \mathrm{~nm}$. Therefore, the modeling approach in this study satisfactorily divulges which spectral shapes arise in the dipolar modes under the Kerker condition specific to the proposed nano shell structure. In other words, MD with 

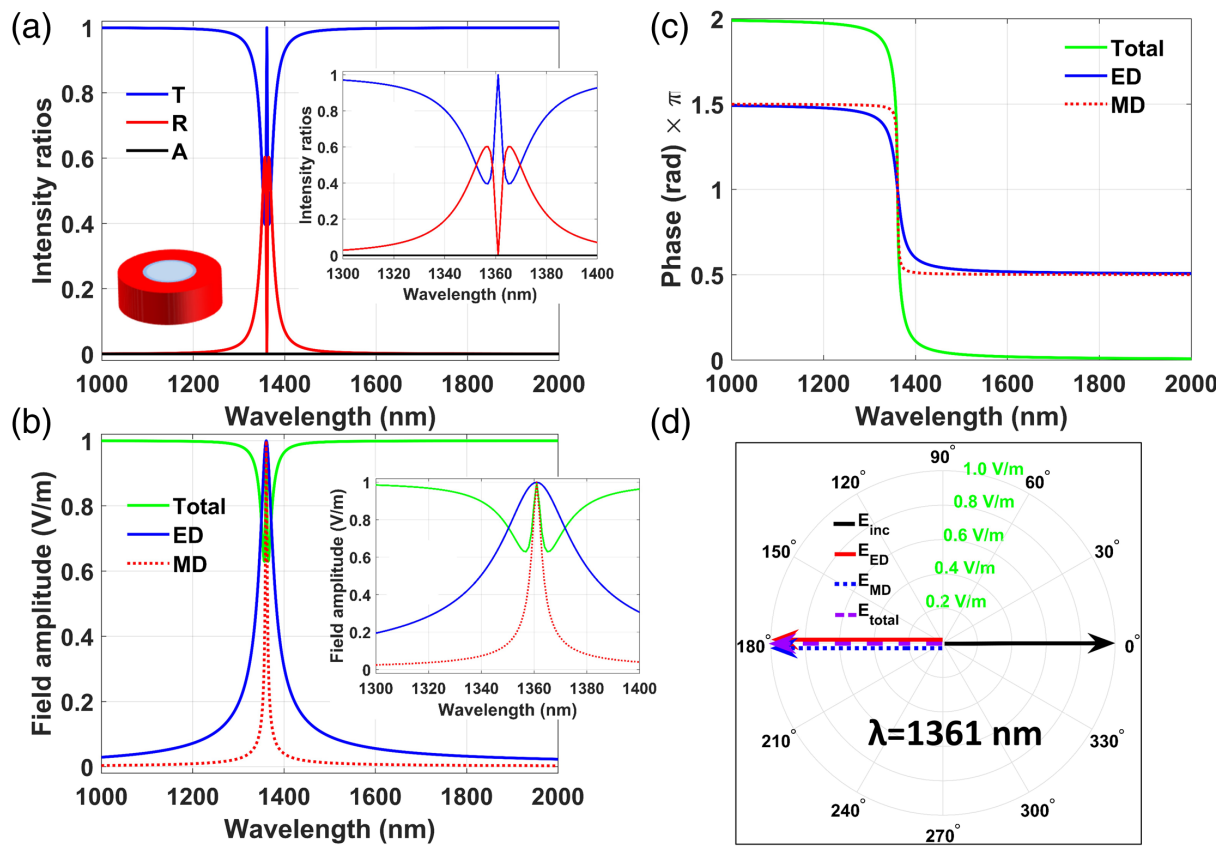

Fig. 8. Analytical results for Case B. (a) Spectral intensity ratios as transmission or transmissivity (blue), reflectivity (red), and absorptivity (black). (b) Electric field amplitude of the transmitted light (green) and the contributions of the electric dipole (blue) and magnetic dipole (red) resonances. (c) Phase spectrum of the transmitted total electric field (green) and the phase spectra of the forward radiated electric field vectors from electric dipole (blue) and magnetic dipole (red) responses. Inset plots in (a) and (b) are zoomed in. (d) Vector diagram displaying the total output electric field vector $\left(E_{\text {total }}\right)$ formed by the interaction of the incident wave $\left(E_{\text {inc }}\right)$ with the forward radiated electric field vectors from electric dipole $\left(E_{\mathrm{ED}}\right)$ and magnetic dipole $\left(E_{\mathrm{MD}}\right)$ resonances at $\lambda=1361 \mathrm{~nm}$.
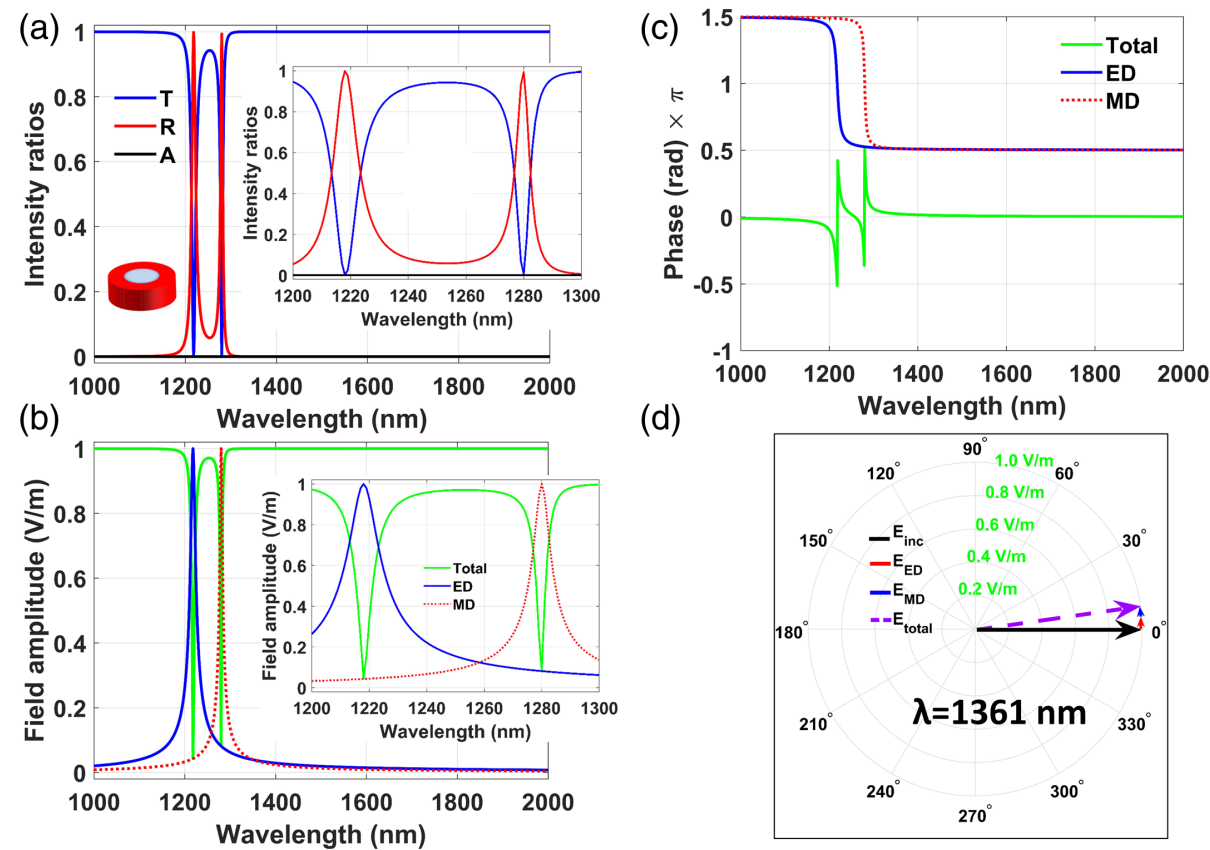

Fig. 9. Analytical results for Case C. (a) Spectral intensity ratios as transmission or transmissivity (blue), reflectivity (red), and absorptivity (black). (b) Electric field amplitude of the transmitted light (green) and the contributions of the electric dipole (blue) and magnetic dipole (red) resonances. (c) Phase spectrum of the transmitted total electric field (green) and the phase spectra of the forward radiated electric field vectors from electric dipole (blue) and magnetic dipole (red) responses. Inset plots in (a) and (b) are zoomed in. (d) Vector diagram displaying the total output electric field vector $\left(E_{\text {total }}\right)$ formed by the interaction of the incident wave $\left(E_{\mathrm{inc}}\right)$ with the forward radiated electric field vectors from electric dipole $\left(E_{\mathrm{ED}}\right)$ and magnetic $\operatorname{dipole}\left(E_{\mathrm{MD}}\right)$ resonances at $\lambda=1361 \mathrm{~nm}$. 
relatively higher- $Q$ outperforms the Kerker state of the nano shell in terms of spectral shape construction in the near vicinity of the overlap frequency $(\lambda=1361 \mathrm{~nm})$. The second feature is seen in Fig. 8(c) that a full $360^{\circ}$ phase coverage is obtained in the phase of the total electric field near the resonance wavelength where the Kerker condition is met, whereas a $180^{\circ}$ phase change occurs in single dipoles.

Finally, the results for Case $\mathrm{C}$ are given in Fig. 9. Similar to Case $\mathrm{A}$, in this case the $\mathrm{ED}$ and $\mathrm{MD}$ resonances are discrete. Differently, ED occurs at smaller wavelengths than MD. Moreover, the linewidths of the resonances are narrower and resonances are closer to each other with respect to Case A. Other similar features and working mechanisms will not be repeated here as they are explained in sufficient detail in Case A. Overall, this work deeply investigates the electric and magnetic polarizabilities of the nano shell meta-atom at the Kerker condition and the two other sides of this special effect. Resonance locations, linewidths, and complete transmission versus reflection with $2 \pi$ phase coverage are enabled by altering the filling factor of the meta-atom.

\section{CONCLUSION}

In this study, an all-dielectric Huygens' metasurface consisting of high-refractive-index nano shells in the low-refractive-index environment is designed and investigated numerically and analytically in the near-infrared spectrum. By simply tuning the nano shell inner radius, the effects on the dipolar resonances are uncovered specific to the proposed design so that one can control the locations of the ED and MD resonances of the nano shell meta-atoms arranged in a periodic array. The type of the dipolar resonances (ED or $\mathrm{MD}$ ) is proven with numerical electromagnetic field distributions. The field distributions for all distinct representative cases possess unique profiles at the $\mathrm{ED}$ and MD wavelengths. In order to better understand the EM wave interactions in the designed Huygens' metasurface, an analytical model based on the coupled discrete dipole approach is applied for the selected three distinct cases of the structure. It is shown that dipolar resonances can be detuned or tuned depending on the inner radius. In the detuned or non-overlapped case, the transmission drops to zero in ED and MD resonances as a result of the destructive interference of the incoming and forward-scattered light. When the ED and MD resonances overlap (Kerker condition), the backscattering is blocked due to the destructive interference in the reverse direction where ED and MD modes are out-of-phase, while the forward scattering is strengthened by the constructive interference of the forwardscattered and in-phase dipolar modes. Moreover, due to the spectral difference between the overlapping dipolar modes in the proposed design, an uneven transparency window around the Kerker point is obtained.

The proposed structure is a thin functional element, wherein so-called metasurfaces can be easily fabricated by standard silicon manufacturing techniques due to its simple design and low aspect ratio. The design and modeling approach in this study can be applied with suitable materials in different optical spectra and even in the RF band. In this way, various beam control components can be developed for a wide range of optical and microwave applications.
Funding. Türkiye Bilimler Akademisi (TUBA).

Acknowledgment. E. Ozbay and H. Kurt acknowledge partial support from the TUBA.

Disclosures. The authors declare no conflicts of interest.

Data Availability. Data underlying the results presented in this paper are not publicly available at this time but may be obtained from the authors upon reasonable request.

\section{REFERENCES}

1. N. Yu, P. Genevet, M. A. Kats, F. Aieta, J.-P. Tetienne, F. Capasso, and Z. Gaburro, "Light propagation with phase reflection and refraction," Science 334, 333-337 (2011).

2. J. Cheng, D. Ansari-Oghol-Beig, and H. Mosallaei, "Wave manipulation with designer dielectric metasurfaces," Opt. Lett. 39, 6285-6288 (2014).

3. M. Decker, I. Staude, M. Falkner, J. Dominguez, D. N. Neshev, I. Brener, T. Pertsch, and Y. S. Kivshar, "High-efficiency dielectric Huygens' surfaces," Adv. Opt. Mater. 3, 813-820 (2015).

4. Z. X. Wang, J. W. Wu, X. Wan, L. W. Wu, Q. Xiao, Q. Cheng, and T. J. Cui, "Broadband and ultrathin Huygens metasurface with high transmittance," J. Phys. D 53, 455102 (2020).

5. E. Ozbay, "Plasmonics: merging photonics and electronics at nanoscale dimensions," Science 311, 189-193 (2006).

6. N. Meinzer, W. L. Barnes, and I. R. Hooper, "Plasmonic meta-atoms and metasurfaces," Nat. Photonics 8, 889-898 (2014).

7. S. Jahani and Z. Jacob, "All-dielectric metamaterials," Nat. Nanotechnol. 11, 23-36 (2016).

8. A. J. Ollanik, J. A. Smith, M. J. Belue, and M. D. Escarra, "Highefficiency all-dielectric Huygens metasurfaces from the ultraviolet to the infrared," ACS Photonics 5, 1351-1358 (2018).

9. A. Ozer, N. Yilmaz, H. Kocer, and H. Kurt, "Polarization-insensitive beam splitters using all-dielectric phase gradient metasurfaces at visible wavelengths," Opt. Lett. 43, 4350-4353 (2018).

10. A. Ozer, N. Yilmaz, H. Kocer, and H. Kurt, "Broadband asymmetric light transmission based on all-dielectric metasurfaces in the visible spectrum," J. Opt. 21, 055104 (2019).

11. X. Qiu, J. Shi, Y. Li, and F. Zhang, "All-dielectric multifunctional transmittance-tunable metasurfaces based on guided-mode resonance and ENZ effect," Nanotechnology 32, 065202 (2021).

12. A. Leitis, A. Heßler, S. Wahl, M. Wuttig, T. Taubner, A. Tittl, and H. Altug, "All-dielectric programmable Huygens' metasurfaces," Adv. Funct. Mater. 30, 1910259 (2020).

13. H. Kocer, Y. Durna, H. Kurt, and E. Ozbay, "Dynamic beam splitter employing an all-dielectric metasurface based on an elastic substrate," Opt. Lett. 45, 3521-3524 (2020).

14. E. Hasman, V. Kleiner, G. Biener, and A. Niv, "Polarization dependent focusing lens by use of quantized Pancharatnam-Berry phase diffractive optics," Appl. Phys. Lett. 82, 328-330 (2003).

15. D. Lin, P. Fan, E. Hasman, and M. L. Brongersma, "Dielectric gradient metasurface optical elements," Science 345, 298-302 (2014).

16. R. C. Devlin, M. Khorasaninejad, W. T. Chen, J. Oh, and F. Capasso, "Broadband high-efficiency dielectric metasurfaces for the visible spectrum," Proc. Natl. Acad. Sci. USA 113, 10473-10478 (2016).

17. F. Capasso, M. Khorasaninejad, W. T. Chen, R. C. Devlin, J. Oh, and A. Y. Zhu, "Metalenses at visible wavelengths: diffraction-limited focusing and subwavelength resolution imaging," Science $\mathbf{3 5 2}$, 1190-1194 (2016).

18. E. Maguid, I. Yulevich, M. Yannai, V. Kleiner, M. L. Brongersma, and E. Hasman, "Multifunctional interleaved geometric-phase dielectric metasurfaces," Light Sci. Appl. 6, e17027 (2017).

19. A. Y. Zhu, A. I. Kuznetsov, B. Luk'yanchuk, N. Engheta, and P. Genevet, "Traditional and emerging materials for optical metasurfaces," Nanophotonics 6, 452-471 (2017).

20. M. Khorasaninejad, A. Y. Zhu, C. Roques-Carmes, W. T. Chen, J. Oh, I. Mishra, R. C. Devlin, and F. Capasso, "Polarization-insensitive metalenses at visible wavelengths," Nano Lett. 16, 7229-7234 (2016).

21. E. Arbabi, A. Arbabi, S. M. Kamali, Y. Horie, and A. Faraon, "High efficiency double-wavelength dielectric metasurface lenses with 
dichroic birefringent meta-atoms," Opt. Express 24, 18468-18477 (2016).

22. Z. Zhou, J. Li, R. Su, B. Yao, H. Fang, K. Li, L. Zhou, J. Liu, D. Stellinga, C. P. Reardon, T. F. Krauss, and X. Wang, "Efficient silicon metasurfaces for visible light," ACS Photonics 4, 544-551 (2017).

23. A. Arbabi, Y. Horie, M. Bagheri, and A. Faraon, "Dielectric metasurfaces for complete control of phase and polarization with subwavelength spatial resolution and high transmission," Nat. Nanotechnol. 10, 937-943 (2015).

24. Y. Tian, Z. Li, Z. Xu, Y. Wei, and F. Wu, "High transmission focusing lenses based on ultrathin all-dielectric Huygens' metasurfaces," Opt. Mater. 109, 110358 (2020)

25. S. Liu, A. Vaskin, S. Campione, O. Wolf, M. B. Sinclair, J. Reno, G. A. Keeler, I. Staude, and I. Brener, "Huygens' metasurfaces enabled by magnetic dipole resonance tuning in split dielectric nanoresonators," Nano Lett. 17, 4297-4303 (2017).

26. I. Staude, A. E. Miroshnichenko, M. Decker, N. T. Fofang, S. Liu, E. Gonzales, J. Dominguez, T. S. Luk, D. N. Neshev, I. Brener, and Y. Kivshar, "Tailoring directional scattering through magnetic and electric resonances in subwavelength silicon nanodisks," ACS Nano 7, 7824-7832 (2013).

27. P. R. Dezert, "Theoretical study of isotropic Huygens particles for metasurfaces," thesis (Université de Bordeaux, 2019).

28. A. E. H. Love, "I. The integration of the equations of propagation of electric waves," Philos. Trans. R. Soc. London A 197, 1-45 (1901).

29. S. A. Schelkunoff, "Some equivalence theorems of electromagnetics and their application to radiation problems," Bell Syst. Tech. J. 15, 92-112 (1936).

30. A. B. Evlyukhin, C. Reinhardt, A. Seidel, B. S. Luk'Yanchuk, and B. N. Chichkov, "Optical response features of Si-nanoparticle arrays," Phys. Rev. B 82, 04540 (2010).

31. A. B. Evlyukhin, C. Reinhardt, and B. N. Chichkov, "Multipole light scattering by nonspherical nanoparticles in the discrete dipole approximation," Phys. Rev. B 84, 235429 (2011).

32. M. Kerker, D.-S. Wang, and C. L. Giles, "Electromagnetic scattering by magnetic spheres," J. Opt. Soc. Am. 73, 765-767 (1983).
33. B. Rolly, B. Stout, and N. Bonod, "Boosting the directivity of optical antennas with magnetic and electric dipolar resonant particles," Opt. Express 20, 20376-20386 (2012).

34. Y. H. Fu, A. I. Kuznetsov, A. E. Miroshnichenko, Y. F. Yu, and B. Luk'yanchuk, "Directional visible light scattering by silicon nanoparticles," Nat. Commun. 4, 1527 (2013).

35. V. E. Babicheva and J. V. Moloney, "Lattice effect influence on the electric and magnetic dipole resonance overlap in a disk array," Nanophotonics 7, 1663-1668 (2018).

36. V. E. Babicheva and A. B. Evlyukhin, "Resonant lattice Kerker effect in metasurfaces with electric and magnetic optical responses," Laser Photonics Rev. 11, 1700132 (2017).

37. M. A. van de Haar, J. van de Groep, B. J. M. Brenny, and A. Polman, "Controlling magnetic and electric dipole modes in hollow silicon nanocylinders," Opt. Express 24, 2047-2064 (2016).

38. V. R. Almeida, Q. Xu, C. A. Barrios, and M. Lipson, "Guiding and confining light in void nanostructure," Opt. Lett. 29, 1209-1211 (2004).

39. V. Chandra and R. Ranjan, "Performance analysis of slot waveguide using aluminum nitride in slot region," Opt. Quantum Electron. 52, 231 (2020).

40. L. Cong and R. Singh, "Spatiotemporal dielectric metasurfaces for unidirectional propagation and reconfigurable steering of terahertz beams," Adv. Mater. 32, 2001418 (2020).

41. S. Liu, M. B. Sinclair, T. S. Mahony, Y. C. Jun, S. Campione, J. Ginn, D. A. Bender, J. R. Wendt, J. F. Ihlefeld, P. G. Clem, J. B. Wright, and I. Brener, "Optical magnetic mirrors without metals," Optica 1, 250-256 (2014).

42. A. S. Schwanecke, V. A. Fedotov, V. V. Khardikov, S. L. Prosvirnin, Y. Chen, and N. I. Zheludev, "Optical magnetic mirrors," J. Opt. A 9, L1 (2007).

43. A. Krishnan, A. B. O'Gorman, and M. L. Povinelli, "Design of switchable, narrowband thermal absorption peaks in metal-vanadium-dioxide gratings," J. Opt. 22, 094002 (2020). 